\title{
STEINER TRIPLE SYSTEMS OF ORDER 19 WITH NONTRIVIAL AUTOMORPHISM GROUP
}

\author{
CHARLES J. COLBOURN, SPYROS S. MAGLIVERAS, AND D. R. STINSON
}

\begin{abstract}
There are 172,248 Steiner triple systems of order 19 having a nontrivial automorphism group. Computational methods suitable for generating these designs are developed. The use of tactical configurations in conjunction with orderly algorithms underlies practical techniques for the generation of the designs, and the subexponential time isomorphism technique for triple systems is improved in practice to test isomorphisms of the designs. The automorphism group of each of the triple systems is computed, and a summary presented of the number of systems with each possible type of automorphism.
\end{abstract}

\section{STEINER TRIPLE SYSTEMS AND THEIR GROUPS}

A Steiner triple system of order $v$, briefly $\operatorname{STS}(v)$, is a pair $(V, \mathscr{B})$, where $V$ is a set of $v$ elements and $\mathscr{B}$ is a set of 3-element subsets of $V$, with the property that every 2 -subset of $V$ appears in exactly one subset of $\mathscr{B}$. Sets in $\mathscr{B}$ are triples. An automorphism of an $\operatorname{STS}(v)$ is a permutation on $V$ that maps each triple in $\mathscr{B}$ to a triple of $\mathscr{B}$, and the automorphism group is the group of all automorphisms of the STS.

Steiner triple systems with nontrivial automorphisms have been studied for many reasons. Not least among these is the fact that Steiner triple systems are too numerous to examine exhaustively, even for order 19. In fact, Stinson and Ferch [20] have shown that the number of nonisomorphic STS(19) is at least $2,395,687$ and estimate the exact number to be on the order of $10^{9}$. For this reason, much effort has been concentrated on STS(19) with additional properties.

Bays [1] enumerated the four STS(19) having a cyclic automorphism in 1932. More recently, Denniston [8] generated the 184 nonisomorphic STS(19) with a reversal, i.e., an automorphism fixing one element and mapping the rest in nine 2-cycles. Phelps and Rosa [13] generated the ten nonisomorphic STS(19) having a 2-rotational automorphism, i.e., an automorphism fixing one element and mapping the remainder in two 9-cycles. Using Gelling's list of 1-factorizations of order ten [9] and properties of their automorphism groups, Stinson and Seah [21] determined that the number of STS(19) having a subsystem of order 9 is precisely 284,457 . See Prince $[14,15]$ for some related results on the enumeration of $\operatorname{STS}(19)$.

Received by the editor February 7, 1991.

1991 Mathematics Subject Classification. Primary 05B07. 
In this paper, we examine all possible nontrivial automorphisms that an STS(19) can admit. We determine a minimum subset of basic automorphisms that must be considered in order to generate all STS(19) having a nontrivial automorphism group. For each basic automorphism, we develop computational methods to generate all nonisomorphic STS(19) having the specified automorphism; finally, we determine the full automorphism group of each. This permits us to determine the total number of nonisomorphic STS(19) having a nontrivial automorphism group, namely 172,248 . We present the computational results in $\S 3$, giving all those systems having group order at least 9 .

Let $\pi$ be a permutation of 19 symbols. We say that $\pi$ has type $a_{1}^{n_{1}} \cdots a_{t}^{n_{t}}$ if $\pi$ contains $n_{i}$ cycles of length $a_{i}$, for $1 \leq i \leq t$. It is easy to see that an appropriate power of $\pi$ has type $1^{n} a^{m}$, where $a$ is prime, for some $n, a$, and $m$ such that $n+a m=19$. Now, the set of fixed points of any automorphism forms a subsystem of the STS(19); hence, $n \in\{0,1,3,7,9\}$.

We can rule out the case $n=9$ as follows. If there existed an automorphism of an STS(19) having nine fixed points forming a subsystem, then deletion of these nine points would yield a one-factorization of $K_{10}$ having an automorphism fixing every one-factor. However, it is not difficult to prove that there exists a one-factorization of $K_{2 n}$ having an automorphism that fixes every onefactor if and only if $n$ is even:

Lemma 1.1. For $n \geq 2$ there exists a 1-factorization of $K_{2 n}$ having a nontrivial automorphism that fixes every factor if and only if $n$ is even.

Proof. Suppose that $F_{1}, F_{2}, \ldots, F_{2 n-1}$ is a 1-factorization of $K_{2 n}, n$ odd, and that $\pi$ is an automorphism that fixes every factor. If $\pi$ has a fixed point $x$, since every factor is fixed, every edge containing $x$ is fixed, and then $\pi$ is the identity automorphism. Otherwise, without loss of generality, $\pi$ has type $t^{u}$ for $t \geq 2$ and $t u=2 n$. Now if $t>2$, consider an orbit $\left(x_{0}, \ldots, x_{t-1}\right)$ of elements under $\pi$. Suppose that $\left\{x_{0}, x_{1}\right\} \in F_{i}$. Then, since $\pi$ is an automorphism, $\left\{x_{1}, x_{2}\right\} \in F_{i}$, and $F_{i}$ is not a 1 -factor; this ensures that $t \leq 2$. Hence, it remains to treat the case when $\pi$ has type $2^{n}$.

Let $\pi=(12)(34) \cdots(2 n-12 n)$. Call edges of the form $\{2 i-1,2 i\}$, $(1 \leq i \leq n)$ inside edges, and the rest outside edges. Every orbit of inside edges under $\pi$ has one, and every orbit of outside edges has size two. Since each factor has an odd number of edges, it has an odd number of inside edges. But there are $2 n-1$ factors and only $n$ inside edges. Thus, for $n \geq 3, \pi$ cannot have type $2^{n}$.

Conversely, suppose $n$ is even and write $n=2 \mathrm{~m}$. We shall construct a 1factorization of $K_{4 m}$ on the vertex set $\left\{\infty, \infty^{\prime}, 0,1, \ldots, 2 m-2,0^{\prime}, 1^{\prime}, \ldots\right.$, $\left.(2 m-2)^{\prime}\right\}$. The one-factors are as follows. First, define $M$ to be the factor $\left\{\left\{\infty, \infty^{\prime}\right\}\right\} \cup\left\{\left\{j, j^{\prime}\right\}: 0 \leq j \leq 2 m-2\right\}$. Next, define factors

$$
\begin{aligned}
F_{0}= & \left\{\{\infty, 0\},\left\{\infty^{\prime}, 0^{\prime}\right\}\right\} \\
& \cup\left\{\{j, 2 m-1-j\},\left\{j^{\prime},(2 m-1-j)^{\prime}\right\}: 0 \leq j \leq m-1\right\}
\end{aligned}
$$

and

$$
\begin{aligned}
G_{0}= & \left\{\left\{\infty, 0^{\prime}\right\},\left\{\infty^{\prime}, 0\right\}\right\} \\
& \left.\cup\left\{j,(2 m-1-j)^{\prime}\right\},\left\{j^{\prime}, 2 m-1-j\right\}: 0 \leq j \leq m-1\right\} .
\end{aligned}
$$


Then, for $1 \leq i \leq 2 m-2$, define $F_{i}$ (respectively $G_{i}$ ) to be the factor $i+F_{0}$ (resp. $\left.i+G_{0}\right)$ developed $\bmod 2 m-1$. It is easy to see that

$$
\mathscr{F}=\{M\} \cup\left\{F_{i}: 0 \leq i \leq 2 m-1\right\} \cup\left\{G_{i}: 0 \leq i \leq 2 m-1\right\}
$$

is a 1 -factorization of $K_{4 m}$.

Since 5 is odd, there is no automorphism of an $\operatorname{STS}(19)$ that fixes nine points. Hence, we restrict our attention to automorphisms of the type $1^{n} a^{m}$, where $n \in\{0,1,3,7\}$ and $a \geq 2$ is prime. Such an automorphism is called a basic automorphism.

Thus, the basic automorphisms that we must consider are those of types $19^{1}, 1^{1} 2^{9}, 1^{1} 3^{6}, 1^{3} 2^{8}, 1^{7} 2^{6}$, and $1^{7} 3^{4}$. The first two are the cyclic and reverse automorphisms treated by Bays [1] and Denniston [8], respectively. The remainder have not previously been treated, although the 2-rotational STS(19) [13] (having an automorphism of type $1^{1} 9^{2}$ ) are a small fraction of those having an automorphism of type $1^{1} 3^{6}$.

To generate all nonisomorphic STS(19) having a nontrivial automorphism group, it suffices to generate all nonisomorphic STS(19) admitting each of the six basic types of automorphisms. In $\S 2$, we discuss methods suitable for this task.

\section{Computational methods}

For a basic automorphism $\pi$, our computational task devolves naturally into two phases: generating a complete list of solutions, and extracting one representative of each isomorphism class. The problems associated with poor isomorph rejection during the generation phase have been discussed extensively (see, for example, [16]), so we do not belabor the issue here. We employed three main methods (carrying out a number of the computations independently, using different methods).

2.1. Tactical decompositions. The primary method we employed proceeds as follows. Suppose that $\pi$ is a permutation, and that we are to generate all STS having $\pi$ as an automorphism. Let $p_{1}, \ldots, p_{s}$ be the point-orbits, and $b_{1}, \ldots, b_{u}$ the block-orbits under $\pi$, of lengths $n_{1}, \ldots, n_{s}$ and $m_{1}, \ldots, m_{u}$, respectively. For $1 \leq j \leq u$, let $B_{j}$ be any block in $b_{j}$. We define the tactical decomposition of the STS with respect to $\pi$ to be the $s \times u$ matrix $T_{\pi}=\left(t_{i j}\right)$, where $t_{i j}=\left|p_{i} \cap B_{j}\right|$. If $r=(v-1) / 2$ is the number of blocks passing through any given point of the STS, it is easy to see that

1. for $1 \leq i \leq s, \sum_{q=1}^{u} t_{i q} m_{q}=r n_{i}$,

2. for $1 \leq i, j \leq s, i \neq j, \sum_{q=1}^{u} t_{i q} t_{j q} m_{q}=n_{i} n_{j}$,

3. for $1 \leq i \leq s, \sum_{q=1}^{u}\left(\begin{array}{c}t_{i q} \\ 2\end{array}\right) m_{q}=\left(\begin{array}{c}n_{i} \\ 2\end{array}\right)$.

It is convenient to view a tactical decomposition of an STS as a collection of $u$ multisets of the elements $\left\{p_{1}, \ldots, p_{s}\right\}$, where the columns of $T_{\pi}=\left(t_{i j}\right)$ are the characteristic vectors of the multisets. In particular, $t_{i j}$ specifies the multiplicity of $p_{i}$ in the $j$ th multiset.

We restrict our attention only to possible basic automorphisms; thus, the order of $\pi$ will be 19,3 , or 2 . We then observe the following.

1. For $1 \leq i \leq s$, if $n_{i}=2$, there is exactly one multiset of the form $\left\{p_{i}, p_{i}, p_{j}\right\}$. Here we must have $n_{j}=1$. This multiset corresponds to a fixed block under $\pi$. 
2. If $n_{i}=3$, then the total number of occurrences of $\left\{p_{i}, p_{i}\right\}$ among the $u$ multisets is three, counting $\left\{p_{i}, p_{i}, p_{i}\right\}$ as three occurrences.

3. If $n_{i}=19$, then the total number of occurrences of $\left\{p_{i}, p_{i}\right\}$ among the multisets is nine, counting $\left\{p_{i}, p_{i}, p_{i}\right\}$ as three occurrences.

Let us consider the tactical decompositions for the basic automorphisms of STS(19) . For $19^{1}$, there is a unique tactical decomposition, having one pointorbit and three multisets of the form $\left\{p_{1}, p_{1}, p_{1}\right\}$. For $1^{1} 2^{9}$, a tactical decomposition has ten point-orbits $p_{1}, \ldots, p_{10}$, with $n_{1}=\cdots=n_{9}=2$ and $n_{10}=1$. It follows from the definition that it has a multiset of the form $\left\{p_{i}, p_{i}, p_{10}\right\}$ for each $1 \leq i \leq 9$, and that it has 24 further multisets of the form $\left\{p_{i}, p_{j}, p_{k}\right\}$ with $1 \leq i<j<k \leq 9$. These 24 multisets must cover every unordered pair $\left\{p_{i}, p_{j}\right\}$ twice, and hence they are the triples of a twofold triple system of order 9. Denniston [8] observes that such a twofold triple system, in order to form the tactical decomposition of an STS(19), cannot have repeated blocks; hence, there are 13 nonisomorphic tactical decompositions to be considered [10].

For type $1^{1} 3^{6}$, we have seven point-orbits $p_{1}, \ldots, p_{7}$, with $n_{1}=\cdots=$ $n_{6}=3$ and $n_{7}=1$. Without loss of generality, we may assume that we have multisets of the form $\left\{p_{2 i-1}, p_{2 i}, p_{7}\right\}$ for $1 \leq i \leq 3$ to dispense with the occurrences of $p_{7}$. Then we may have 0,3 , and 6 occurrences of multisets of the form $\left\{p_{i}, p_{i}, p_{i}\right\}$. In addition, every pair $\left\{p_{i}, p_{j}\right\}$ with $i \neq j$ must be covered three times in total. We generated all tactical decompositions using an orderly algorithm (see $\S 2.3$ later), and used a naive 6 ! algorithm to determine the isomorphism of the resulting decompositions. There are 244 tactical decompositions in this case.

For type $1^{3} 2^{8}$, a tactical decomposition has 11 point-orbits $p_{1}, \ldots, p_{11}$, with $n_{1}=\cdots=n_{8}=2$ and $n_{9}=n_{10}=n_{11}=1$. The occurrences of $p_{9}$, $p_{10}$, and $p_{11}$ exhaust all pairs of the form $\left\{p_{i}, p_{i}\right\}$, along with a collection of pairs on $\left\{p_{1}, \ldots, p_{8}\right\}$ that has each element in two pairs of the collection. So the multisets of the form $\left\{p_{i}, p_{j}, p_{k}\right\}$ with $1 \leq i<j<k \leq 8$ form a partial twofold triple system $\mathscr{T}$ with 2-regular leave (see [3] for information on leaves). Now, if $\mathscr{T}$ has a repeated block $\left\{p_{i}, p_{j}, p_{k}\right\}$, any $\operatorname{STS}(19)$ leading to the tactical decomposition must have six point-orbits carrying four triples of which two are disjoint, which cannot exist; so we may assume that $\mathscr{T}$ is simple. We did not generate the entire tactical decomposition here, but rather just generated the portion $\mathscr{T}$, that is, simple partial twofold triple systems of order 8 with 2-regular leave. For this purpose, it suffices to consider the isomorphism type of the partial twofold triple systems obtained by dropping each point in turn from each of the 36 nonisomorphic twofold triple systems of order 9 [10]. Then we used McKay's isomorphism program nauty [11] to determine that there are 85 nonisomorphic simple partial twofold triple systems of order eight with 2regular leave.

For type $1^{7} 3^{4}$, a tactical decomposition has eleven point-orbits $p_{1}, \ldots, p_{11}$, with $n_{1}=n_{2}=n_{3}=n_{4}=3$ and $n_{5}=\cdots=n_{11}=1$. Since there are 57 triples in an STS(19), and seven appear on the seven fixed points, it follows that there must be precisely two multisets of the form $\left\{p_{i}, p_{i}, p_{i}\right\}$ in a tactical decomposition. In fact, in this case, it is easy to generate all partial triple systems on 19 points with an automorphism of type $1^{7} 3^{4}$ that cover all pairs except those lying on the fixed points; there are ten nonisomorphic such partial triple systems of order 19. 
For the last case, of type $1^{7} 2^{6}$, we examined that portion of the tactical decomposition containing the multisets having at least two of their point orbits representing the orbits of size two. Using a brute-force 6! isomorphism algorithm to eliminate duplicates this led to 82 nonisomorphic partial tactical decompositions.

Once the tactical decompositions are found, there remains the problem of "filling" or "covering" the tactical decomposition to obtain all nonisomorphic STS(19) having the given tactical decomposition. Formally, given a tactical decomposition, we construct a set of elements $\left\{v_{i j}: 1 \leq i \leq s, 0 \leq j<n_{i}\right\}$. Then for each multiset $\left\{p_{i}, p_{j}, p_{k}\right\}$ in turn, and for each $0 \leq a<n_{i}, 0 \leq b<$ $n_{j}, 0 \leq c<n_{k}$, we choose $\left\{v_{i a}, v_{j b}, v_{k c}\right\}$ as the orbit representative of the multiset. This gives a set of orbit representatives (actually a large number of them in general) for an STS(19) having the specified tactical decomposition.

Some isomorph rejection is easily introduced in this covering procedure; particularly, we may choose arbitrarily one of $a, b$, or $c$ to be 0 . If $i=j$, we may take $a=0$ and $b \leq n_{i} / 2$; and if $i=j=k$, we may take $a=0$, $b \leq n_{i}-c, 2 b \leq c$. It is also possible to use the automorphisms of the partial STS constructed so far to determine that other distinct choices lead to isomorphic solutions; in general we did not use the automorphisms of the system as it was built, excepting of course the automorphism used in forming the tactical decomposition, which must be preserved.

Some other general observations are useful here. On the fixed points we must place a subsystem. We have $0,1,3$, or 7 fixed points; there is but one way to place a subsystem of order 1 or 3 . There are 30 distinct ways to place a subsystem of order 7 . Our strategy was to leave the subsystem unspecified until the very last step, and to determine the Steiner triple system with a hole in all nonisomorphic ways. Thus, we elected to fill partial tactical decompositions, omitting the multisets on the fixed points; in the case of $1^{3} 2^{8}$, we went further and omitted all multisets involving the fixed points.

For $1^{7} 3^{4}$, as we remarked earlier, we obtained ten nonisomorphic partial STS(19) having a 7-hole on the fixed points. For $1^{7} 2^{6}$, the 82 tactical decompositions led to 2,729 partial STS(19) having a 7-hole on the fixed points. For $1^{3} 2^{8}$, since we omitted the multisets containing the three fixed points, filling the partial tactical decompositions leads to partial Steiner triple systems of order 16 having an automorphism of type $2^{8}$ and having 3-regular leave. The number of nonisomorphic such systems was found to be 1,832 . All of these isomorphism computations were done using nauty [11].

In all cases but $1^{3} 2^{8}$, it is routine at this point to find a complete list of all STS(19) with the desired automorphism. For the remaining case, we determined by a simple backtracking algorithm all ways to partition the 3-regular leaves into three 1 -factors in such a way that the automorphism of type $2^{8}$ is preserved. Then adding three fixed points, forming triples with the three 1factors, and placing a triple on the three fixed points, gives a complete list of all of the systems.

Unfortunately, filling a tactical decomposition, or filling a hole in a partial system in all possible ways, may lead to duplication in the STS that result. Until this point, we have resorted to a brute-force isomorphism test for very small problems, and to nauty for more substantial ones. However, in cases $1^{3} 2^{8}$ and 
$1^{7} 2^{6}$, there remain approximately 90,000 STS for which isomorphism must be checked. Hence we required a very fast method to determine isomorphism.

2.2. Testing isomorphism of STS. In this section, we give a short description of Miller's algorithm for testing isomorphisms of STS $n$ ) [12]. This algorithm has also been studied in [4, 19,7], and has been found to be quite fast for practical isomorphism testing. We first outline the algorithm, and then describe some improvements.

Let $\mathbf{X}$ be the $n$-set $\{1,2, \ldots, n\}$. Let $A=\left\{x_{1}, y_{1}, z_{1}\right\}$ and $B=\left\{x_{2}, y_{2}\right.$, $\left.z_{2}\right\}$ be two 3-subsets of $\mathbf{X}$, where $x_{1}<y_{1}<z_{1}$ and $x_{2}<y_{2}<z_{2}$. We define a lexicographical ordering on the 3-subsets of $\mathbf{X}$ by saying that $A<B$ if $y_{1}<y_{2}$; or $y_{1}=y_{2}$ and $x_{1}<x_{2}$; or $y_{1}=y_{2}, x_{1}=x_{2}$, and $z_{1}<z_{2}$ (note that this is a different lexicographical ordering than was used in $[19,7])$. Now, let $\left(\mathbf{X}, \mathscr{A}_{1}\right)$ and $\left(\mathbf{X}, \mathscr{A}_{2}\right)$ be two $\operatorname{STS}(n)$. Denote $\mathscr{A}_{1}=\left\{A_{11}, \ldots, A_{1 b}\right\}$ and $\mathscr{A}_{2}=\left\{A_{21}, \ldots, A_{2 b}\right\}$, where $b=n(n-1) / 6, A_{11}<A_{12}<\cdots<A_{1 b}$, and $A_{21}<A_{22}<\cdots<A_{2 b}$. Then, define $\mathscr{A}_{1}<\mathscr{A}_{2}$ if there exists an integer $j$ $(1 \leq j \leq b)$ such that $A_{1 i}=A_{2 i}$ for $1 \leq i<j$, and $A_{1 j}<A_{2 j}$.

Suppose $(\mathbf{X}, \mathscr{A})$ is an $\operatorname{STS}(n)$ and $\pi$ is a permutation of $\mathbf{X}$. Define $A^{\pi}=$ $\left\{x^{\pi}: x \in A\right\}$ for $A \in \mathscr{A}$, and $\mathscr{A}^{\pi}=\left\{A^{\pi}: A \in \mathscr{A}\right\}$. We say that $(\mathbf{X}, \mathscr{A})$ is canonical if $\mathscr{A} \leq \mathscr{A}^{\pi}$ for every permutation $\pi$. The canonical form of the STS $(\mathbf{X}, \mathscr{A})$ is the (unique) STS $\left(\mathbf{X}, \mathscr{A}^{\pi}\right)$ that is canonical. It is easy to see that two $\operatorname{STS}(n)$ on the same point set, say $\left(\mathbf{X}, \mathscr{A}_{1}\right)$ and $\left(\mathbf{X}, \mathscr{A}_{2}\right)$, are isomorphic if and only if they have the same canonical forms.

Let $1 \leq m \leq n$. Define a partial labelling of rank $m$ to be a partial permutation $\theta$ of $\{1,2, \ldots, n\}$ such that $|\operatorname{dom}(\theta)|=m$ and $\left\{x^{\theta}: x \in \operatorname{dom}(\theta)\right\}=$ $\{1,2, \ldots, m\}$. A point $x \in \operatorname{dom}(\theta)$ is said to be labelled. If $A$ is a block containing precisely two labelled points, say $x$ and $y$, define $A^{\theta^{\prime}}=\left\{x^{\theta}, y^{\theta}\right\}$.

The algorithm iterates two steps, called "forcing" and "choosing," until all elements have been labelled. Suppose $\theta$ is a partial labelling of rank $m$, and suppose there exists at least one block containing exactly two labelled points. Of these blocks, let $M(\theta)$ denote the block $A$ such that $A^{\theta^{\prime}}$ is lexicographically least, and let $z$ be the unlabelled point in $A$. Suppose $\pi$ is any permutation that extends $\theta$, and suppose that $\left(\mathbf{X}, \mathscr{A}^{\pi}\right)$ is the canonical form of $(\mathbf{X}, \mathscr{A})$. Then, it is easy to see that $z^{\pi}=m+1$. This operation is called "forcing."

When a forcing operation cannot be done, it must be the case that the labelled points form a subsystem of the original $\operatorname{STS}(n)$. Whenever this happens, we take each unlabelled point $z$ in turn, assign $z^{\theta}=m+1$, and then applying forcing operations. This is called a "choosing" operation.

A labelling produced by a succession of "forcing" and "choosing" operations, as described above, is called a candidate labelling. The number of candidate labellings is $O\left(n^{\log n}\right)$, a significant improvement over $O(n !)$.

Even though we are unable to improve the asymptotic running time, we have some further improvements, which speed up isomorphism testing in practice. First among these observations is that we can often reject a partial labelling, $\theta$, as the algorithm is executing. Suppose $\pi$ denotes the "best" candidate labelling at any stage during the execution of the algorithm. Then, at any time in the algorithm, we can compare the partial system consisting of all $A^{\theta}$ such that $A$ is a labelled block to the corresponding set of blocks in $\mathscr{A}^{\pi}$, and abandon 
all extensions of $\theta$ if the partial system is already lexicographically larger than that in $\mathscr{A}^{\pi}$.

The second observation leads to a further restriction on the number of labellings that need to be considered. For a given candidate labelling $\pi$, define $r(\pi)$ to be the number of "choosing" steps that were made in producing $\pi$, and define $s(\pi)=\left(s_{1}, \ldots, s_{r}\right)$ to be the sequence of values $m$ for which the symbols $\{1, \ldots, m\}$ induce a subsystem of $\mathscr{A}^{\pi}$. In the case $n=19$, the possible sequences that can occur are $(0,1,3,19),(0,1,3,7,19)$, and $(0,1,3,9,19)$. Suppose we consider only those candidate labellings $\pi$ for which

1. $r(\pi)$ is minimum, and

2. $s(\pi)$ is lexicographically minimum among all candidate labellings for which $r(\pi)$ is minimum.

Denote the set of candidate labellings satisfying the above two conditions by $\Pi(\mathscr{A})$. Then it is not difficult to show that, if $\left(\mathbf{X}, \mathscr{A}_{1}\right)$ and $\left(\mathbf{X}, \mathscr{A}_{2}\right)$ are isomorphic, then

$$
\left\{\mathscr{A}_{1}^{\pi}: \pi \in \Pi\left(\mathscr{A}_{1}\right)\right\}=\left\{\mathscr{A}_{2}^{\pi}: \pi \in \Pi\left(\mathscr{A}_{2}\right)\right\} .
$$

Also, if $\left(\mathbf{X}, \mathscr{A}_{1}\right)$ and $\left(\mathbf{X}, \mathscr{A}_{2}\right)$ are not isomorphic, then the two sets are disjoint. This means that we can define the canonical form of an $\operatorname{STS}(n),\left(\mathbf{X}, \mathscr{A}_{2}\right)$, relative to the candidate numberings in $\Pi(\mathscr{A})$ (but observe that this may not yield the same canonical form as was defined earlier).

Given any STS(19), we can show that there must exist at least one candidate labelling $\pi$ such that $r(\pi)=4$ and hence $s(\pi)=(0,1,3,19)$. Consequently, we can abandon any partial labelling as soon as any subsystem of order 7 or 9 is generated. This is a result of the nonexistence of a Steiner space of order 19 [22].

We used this algorithm to compute the canonical forms and automorphism groups for over 200,000 systems that were generated in the course of the computations. Our experience is that, except for the two designs with the largest automorphism groups, this method outperformed nauty applied to the elementblock incidence graph. When the system has a group of order 2, our labelling algorithm was an order of magnitude faster. In fairness to the general program nauty, however, we remark that we did no initial partitioning of the triples using isomorphism invariants, and this would surely have accelerated its computation.

2.3. Orderly algorithms. In essence, the use of tactical decompositions employs isomorph rejection at four stages: some isomorph rejection in generating tactical decompositions, complete isomorph elimination on the resulting tactical decompositions, some isomorph rejection in filling the decompositions to form STS(19), and complete isomorph elimination on the resulting STS(19). There is a tradeoff between the amount of effort invested in isomorph rejection during generation, and isomorph elimination of the resulting configurations.

Read [16] proposed a class of orderly algorithms that do complete isomorph rejection during the generation, and hence obviate the need for any isomorph elimination thereafter. His key idea is to generate objects in some canonical form, and to retain during the generation only those partial configurations that lead to canonical forms. See [16,6] for more information an orderly algorithms for graphs, and [17, 18] for more information on orderly algorithms on designs. 
We employed orderly algorithms in the generation of tactical decompositions; but for generating STS(19) directly, we found the methods to be slower than the approach using tactical decompositions for the basic automorphisms $1^{1} 3^{6}$, $1^{3} 2^{8}$, and $1^{7} 2^{6}$. Our experience is at variance with the observed improvement obtained in generating graphs using orderly algorithms [2]. The reasons are not entirely clear. However, one cause seems evident. In generating graphs by adding an edge, the result is always a graph. However, adding an orbit of triples to a portion of an $\operatorname{STS}(v)$, the result need not be a portion of an $\operatorname{STS}(v)$ at allit may have no completion to an $\operatorname{STS}(v)$. Thus an orderly algorithm may invest a substantial amount of time checking canonicity for partial configurations that cannot be extended to obtain solutions; and it may retain many canonical partial configurations that cannot be extended to solutions. The essential contribution in using tactical decompositions is that many of these nonextendable partial configurations do not arise from any tactical decomposition; hence the initial effort to generate all tactical decompositions can be seen as an effort to curtail the search by eliminating large classes of nonextendable partial solutions.

As we have remarked, orderly algorithms can also be used in conjunction with tactical decompositions (both in generating and in filling them). For filling tactical decompositions, however, we did not employ orderly techniques. The reason is simple: we did not find a canonicity check whose computation for partial systems was competitive with the algorithm of $\S 2.2$ for full systems, and hence the canonicity check incurred more computational expense during filling than it saved during elimination. There may be either a better strategy for filling a tactical decomposition, or a more computationally practical canonicity check, but we did not explore this.

2.4. An algebraic approach. Colbourn, Magliveras, and Mathon [5] generated all $\operatorname{STS}(27)$ with transitive automorphism group; their strategy generates $\operatorname{STS}(v)$ with a given group directly, using extensive but not complete isomorph rejection, followed by complete isomorph elimination. We examine a similar strategy here.

Let $A_{23}$ be the $\left(\begin{array}{c}19 \\ 2\end{array}\right) \times\left(\begin{array}{c}19 \\ 3\end{array}\right)(0,1)$-matrix with rows indexed by unordered pairs, and columns indexed by unordered triples on a 19-set, and having $A_{23}(i, j)=1$ if the $i$ th pair occurs in the $j$ th triple, and 0 otherwise. A $(0,1)$-solution of the matrix equation $A_{23} U=\underline{1}$ gives the characteristic vector of an $\operatorname{STS}(19)$.

Now let $A=A_{23}(\Gamma)$, for a permutation group $\Gamma$ on 19 symbols, be the matrix whose rows are indexed by orbits of pairs under $\Gamma$, and whose columns are indexed by orbits of triples under $\Gamma . A(i, j)$ is the number of times that a fixed pair in the $i$ th orbit of pairs appears in any triple of the $j$ th orbit of triples. Reduce $A$ by removing all of those columns that contain an entry larger than 1 -such an orbit of triples can surely not appear in an STS (19) having group $\Gamma$. Then a $\{0,1\}$-solution to the matrix equation $A U=1$ gives the characteristic vector $U$ of a set of orbits of triples under $\Gamma$ whose union is an STS(19) whose automorphism group contains $\Gamma$. Some immediate isomorph rejection is possible. As noted in [5], applying a permutation $\pi$ in the normalizer of $\Gamma$ in the symmetric group on 19 letters to an STS(19) carries the STS to an isomorphic, but possibly distinct, STS. Since we are interested only in nonisomorphic solutions, we consider the action of the normalizer on the orbits 
of triples under $\Gamma$ (the column indices of $A$ ). Choose a representative for each orbit of columns under the normalizer. Then we can modify $A$ so that for one fixed row, say the first, a 1 entry appears only in a column that is the representative of its normalizer orbit of columns. The solution of $A U=1$ is then a binary knapsack problem (see [5]).

We used this strategy in cases where the group $\Gamma$ has order at least 8 , verifying the results obtained using tactical decompositions. However, for smaller group orders, the isomorph rejection in this approach seems to be too weak, causing millions of distinct STS to be generated. The reason is perhaps clear: after a selection of orbit of triples is made to cover the orbit of pairs indexing the first row, there is a large number of selections remaining for the remaining rows. In the normalizer, the stabilizer of the chosen orbit may map the remaining columns in nontrivial orbits; we are treating each column in such an orbit as being different, but isomorphic STS will result from their selection. The use of the normalizer at every step in the computation merits investigation. Nevertheless, this approach has the same drawback as orderly methods in incorporating such strong isomorph rejection, in that substantial effort may be invested on partial solutions that lead to no STS.

\section{STS(19) WITH NONTRIVIAL GROUPS}

For each basic automorphism type, we generated all nonisomorphic Steiner triple systems of order 19 having at least one automorphism of that type, using the methods outlined in $\S 2$. For the six basic automorphism types, we found the following numbers of nonisomorphic designs:

$\begin{array}{rr}19^{1} & 4 \\ 1^{1} 2^{9} & 184 \\ 1^{1} 3^{6} & 12,021 \\ 1^{3} 2^{8} & 80,591 \\ 1^{7} 2^{6} & 80,558 \\ 1^{7} 3^{4} & 124\end{array}$

Naturally, a number of the systems admit more than one of the basic automorphism types; we used the methods of $\S 2.2$ both to compute the full automorphism group of each system, and to determine a canonical form for each system. For group orders at least four, we verified the computations using nauty. Using the canonical forms, we determined the basic automorphism types admitted by each system (by checking for inclusion of the canonical form of each system with one automorphism type on the list of canonical forms of systems with a different automorphism type).

We summarize the numbers of STS(19) in Table 1 as follows. First we partition the systems according to the order of their automorphism group. Then within each group order, we partition the systems into classes according to the subset of the basic automorphism types that the system admits; when more than one subset occurs, we denote the classes by letters $a, b, c, \ldots$ to distinguish them for the subsequent discussion. For each such subset that arises, we report the number of STS(19) with the specified order of automorphism group and the specified subset of basic automorphism types.

Given the full automorphism groups of these systems, we determined for each the types of all automorphisms of the systems; this permits us to determine the 
TABLE 1. Basic automorphisms

\begin{tabular}{|c|c|c|c|c|c|c|c|c|}
\hline Order & Class & $19^{1}$ & $1^{1} 2^{9}$ & $1^{1} 3^{6}$ & $1^{8} 2^{8}$ & $1^{7} 2^{6}$ & $1^{7} 3^{4}$ & Number of $S T S$ \\
\hline 432 & & & & $\star$ & $\star$ & $\star$ & $\star$ & 1 \\
\hline 171 & & $\star$ & & $\star$ & & & & $\overline{1}$ \\
\hline 144 & & & & $\star$ & $\star$ & $\star$ & & 1 \\
\hline 108 & & & & $\star$ & $\star$ & $\star$ & $\star$ & 1 \\
\hline 96 & & & & $\star$ & $\star$ & $\star$ & & 1 \\
\hline 57 & & $\star$ & & $\star$ & & & & 2 \\
\hline 54 & & & & $\star$ & - & $\star$ & $\star$ & 2 \\
\hline 32 & & & & & $\star$ & $\star$ & & 3 \\
\hline 24 & & & & $\star$ & $\star$ & $\star$ & & 11 \\
\hline 19 & & $\star$ & & & & & & 1 \\
\hline 18 & $\begin{array}{l}\mathbf{a} \\
\mathbf{b} \\
\mathrm{c} \\
\mathrm{d}\end{array}$ & & $\star$ & $\begin{array}{l}\star \\
\star \\
\star \\
\star\end{array}$ & $\star$ & $\begin{array}{l}\star \\
\star\end{array}$ & $\begin{array}{l}\star \\
\star\end{array}$ & $\begin{array}{l}1 \\
2 \\
6 \\
2\end{array}$ \\
\hline 16 & & & & & $\star$ & $\star$ & & 13 \\
\hline 12 & $\begin{array}{l}\text { a } \\
\mathbf{b} \\
\mathrm{c} \\
\mathrm{d}\end{array}$ & & & $\begin{array}{l}\star \star \\
\star \\
\star\end{array}$ & $\begin{array}{l}\star \\
\star \\
\star\end{array}$ & $\begin{array}{l}\star \\
\star \\
\star\end{array}$ & $\star$ & $\begin{array}{r}8 \\
7 \\
12 \\
10\end{array}$ \\
\hline 9 & & & & $\star$ & & & & 19 \\
\hline 8 & $\begin{array}{l}\mathbf{a} \\
\mathbf{b}\end{array}$ & & & & $\begin{array}{l}\star \\
\star \\
\star\end{array}$ & $\star$ & & $\begin{array}{l}84 \\
17\end{array}$ \\
\hline 6 & $\begin{array}{l}\mathbf{a} \\
\mathbf{b} \\
\mathbf{c} \\
\mathbf{d} \\
\mathbf{e}\end{array}$ & & $\star$ & $\begin{array}{l}\star \\
\star \\
\star\end{array}$ & $\begin{array}{l}\star \\
\star\end{array}$ & 太 & $\begin{array}{l}\star \\
\star\end{array}$ & $\begin{array}{r}14 \\
14 \\
116 \\
10 \\
28\end{array}$ \\
\hline $\begin{array}{l}4 \\
3\end{array}$ & $\begin{array}{l}a \\
b \\
c \\
a\end{array}$ & & & $\star$ & $\begin{array}{l}\star \\
\star\end{array}$ & $\begin{array}{l}\star \\
\star\end{array}$ & & $\begin{array}{r}839 \\
662 \\
620 \\
11800\end{array}$ \\
\hline & b & & & & & & $\star$ & 64 \\
\hline 2 & $\begin{array}{l}\text { a } \\
\mathbf{b} \\
\mathbf{c}\end{array}$ & & $\star$ & & $\star$ & $\star$ & & $\begin{array}{r}169 \\
78907 \\
78800\end{array}$ \\
\hline totals & & 4 & 184 & 12021 & 80591 & 80558 & 124 & 172248 \\
\hline
\end{tabular}

number of STS(19) with every possible type of nontrivial automorphism. Of course, for the basic automorphisms, this information is given in Table 1. In Table 2, we give the same information for the nonbasic automorphisms. We use the partitioning of Table 1 again in Table 2; thus we omit the information about basic automorphisms, since this can be easily determined by looking in Table 1 under the appropriate group order and class. Nevertheless, we remark that although two STS(19) may have the same group order and class (same subset of the basic automorphisms), they need not have the same subset of nonbasic automorphisms. Hence, some classes of Table 1 partition further in Table 2. We omit those systems whose group is generated by a single basic automorphism. 
TABLE 2. Nonbasic automorphisms

\begin{tabular}{|c|c|c|c|c|c|c|c|c|c|c|}
\hline Class & $1^{1} 9^{2}$ & $1^{1} 6^{3}$ & $1^{1} 3^{2} 6^{2}$ & $1^{1} 2^{1} 4^{4}$ & $1^{1} 2^{1} 8^{2}$ & $1^{3} 8^{2}$ & $1^{3} 4^{4}$ & $1^{3} 2^{2} 6^{2}$ & $1^{3} 2^{2} 4^{3}$ & $\#$ \\
\hline 432 & & & $\star$ & & & $\star$ & $\star$ & $\star$ & & 1 \\
\hline 171 & $\star$ & & & & & & & & & 1 \\
\hline$\overline{144}$ & & & $\star$ & $\star$ & $\star$ & & $\star$ & & & 1 \\
\hline 108 & & & $\star$ & & & & $\star$ & & & 1 \\
\hline 96 & & & $\star$ & & & & & $\star$ & & 1 \\
\hline 57 & & & & & & & & & & 2 \\
\hline 54 & & & $\star$ & & & & & & & 2 \\
\hline 32 & & & & $\star$ & & & $\star$ & & & 3 \\
\hline 24 & & & $\star$ & & & & & & & 11 \\
\hline $18 \mathrm{a}$ & & $\star$ & & & & & & & & 1 \\
\hline $18 \mathrm{~b}$ & & & $\star$ & & & & & & & 2 \\
\hline $18 \mathrm{c}$ & & & $\star$ & & & & & & & 6 \\
\hline $18 d$ & & & & & & & & $\star$ & & 2 \\
\hline 16 & & & & & & $\star$ & $\star$ & & & 1 \\
\hline & & & & $\star$ & $\star$ & & $\begin{array}{l}\star \\
\star\end{array}$ & & & $\begin{array}{l}1 \\
5\end{array}$ \\
\hline & & & & $\star$ & & & & & & 6 \\
\hline $12 a$ & & & $\star$ & & & & & & & 8 \\
\hline $12 b$ & & & & & & & & & & 7 \\
\hline $12 c$ & & & & & & & & & & 12 \\
\hline $12 \mathrm{~d}$ & & & & & & & & $\star$ & & 10 \\
\hline 9 & $\star$ & & & & & & & & & 9 \\
\hline $8 a$ & & & & & & & & & & $\frac{10}{84}$ \\
\hline $8 b$ & & & & & $\star$ & & $\star$ & & & 10 \\
\hline & & & & $\star$ & & $\star$ & $\begin{array}{l}\star \\
\star\end{array}$ & & & $\begin{array}{l}\mathbf{5} \\
\mathbf{2}\end{array}$ \\
\hline 68 & & $\star$ & & & & & & & & 14 \\
\hline $6 \mathrm{~b}$ & & & & & & & & & & 14 \\
\hline $6 c$ & & & $\star$ & & & & & & & $\begin{array}{r}104 \\
12\end{array}$ \\
\hline $6 d$ & & & & & & & & $\star$ & & 10 \\
\hline $6 e$ & & & & & & & & & & 28 \\
\hline $4 a$ & & & & & & & & & & 839 \\
\hline $4 b$ & & & & $\star$ & & & 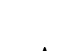 & & & 498 \\
\hline & & & & & & & & & & 11 \\
\hline $4 c$ & & & & & & & & & $\star$ & 48 \\
\hline totals & 10 & 15 & 137 & 518 & 16 & 4 & 183 & 24 & 48 & \\
\hline
\end{tabular}

Any type of automorphism not explicitly mentioned in Tables 1 or 2 does not arise as the type of an automorphism of an STS(19). In particular, all automorphisms of STS(19) have order $1,2,3,4,6,8,9$, or 19. We remark that the number of STS(19) having an automorphism of type $1^{1} 9^{2}$ is ten, in agreement with Phelps and Rosa [13]; and the number of 3-rotational STS(19) (type $1^{1} 6^{3}$ ) is fifteen, in agreement with Denniston [8]. Using the algebraic approach of $\S 2.4$, we generated independently all systems whose group contains 
$Z_{9}, Z_{3} \times Z_{3}$, or $Z_{8}$, verifying in the process the number of STS(19) with an automorphism of type $1^{1} 9^{2}, 1^{3} 8^{2}$, and $1^{1} 2^{1} 8^{2}$.

Naturally, it is not reasonable to list all 172,248 systems on paper. In the supplement at the end of this issue, we list only those having a group of order at least 9 (104 nonisomorphic designs).

It does not appear to be feasible at the present time to get an exact count of STS(19) with trivial group, in order to complete the classification of STS(19).

\section{ACKNOWLEDGMENTS}

Research of the first and third authors is supported by NSERC Canada under grants A0579 and A9287. Research of the second author is supported under NSA grant 88F-066. Research of the second and third authors is supported by the Center for Communication and Information Science, UNL.

\section{BIBLIOGRAPHY}

1. S. Bays, Sur les systèmes cycliques de triples de Steiner différent pour n premier (ou puissance de nombre premier) de la forme $6 n+1$, Comment. Math. Helv. 4 (1932), 183-194.

2. R. D. Cameron, C. J. Colbourn, R. C. Read, and N. C. Wormald, Cataloguing the graphs on 10 vertices, J. Graph Theory 9 (1985), 551-562.

3. C. J. Colbourn, Leaves, excesses and neighbourhoods, Acta Univ. Carolin.-Math. Phys. 28 (1987), 41-47.

4. C. J. Colbourn and M. J. Colbourn, Combinatorial isomorphism problems involving 1factorizations, Ars Combin. 9 (1980), 191-200.

5. C. J. Colbourn, S. S. Magliveras, and R. A. Mathon, Transitive Steiner and Kirkman triple systems of order 27, Math. Comp. 58 (1992), 441-450.

6. C. J. Colbourn and R. C. Read, Orderly algorithms for graph generation, Internat. J. Comput. Math. A7 (1979), 167-172.

7. C. J. Colbourn, D. R. Stinson, and L. Teirlinck, A parallelization of Miller's $n^{\log n}$ isomorphism algorithm (submitted).

8. R. H. F. Denniston, Nonisomorphic reverse Steiner triple systems of order 19, Ann. Discrete Math. 7 (1980), 255-264.

9. E. N. Gelling and R. E. Odeh, On 1-factorizations of the complete graph and the relation to round-robin schedules, Congr. Numer. 9 (1974), 213-221.

10. R. A. Mathon and A. Rosa, A census of Mendelsohn triple systems of order nine, Ars Combin. 4 (1977), 309-315.

11. B. D. McKay, Nauty user's guide (version 1.5), Technical Report TR-CS-90-02, Computer Science Department, Australian National University, 1990.

12. G. L. Miller, On the $n^{\log n}$ isomorphism technique, Proc. Tenth Annual ACM Sympos. Theory of Computing, ACM, New York, 1978, pp. 51-58.

13. K. Phelps and A. Rosa, Steiner triple systems with rotational automorphisms, Discrete Math. 33 (1981), 57-66.

14. A. R. Prince, Steiner triple systems of order 19 constructed from the Steiner triple system of order 9, Proc. Roy. Soc. Edinburgh Sect. A 94 (1983), 89-92.

15. Steiner triple systems of order 19 associated with a certain type of projective plane of order 10, Period. Math. Hungar. 17 (1986), 177-184.

16. R. C. Read, Every one a winner, Ann. Discrete Math. 2 (1978), 107-120.

17. E. Seah, On the enumeration of Howell designs and 1-factorizations using orderly algorithms, Ph.D. Thesis, Department of Computer Science, University of Manitoba, 1987.

18. E. Seah and D. R. Stinson, On the enumeration of one-factorizations of complete graphs containing prescribed automorphism groups, Math. Comp. 50 (1988), 607-618. 
19. D. R. Stinson, Isomorphism testing of Steiner triple systems: canonical forms, Ars Combin. 19 (1985), 213-218.

20. D. R. Stinson and H. Ferch, 2000000 Steiner triple systems of order 19, Math. Comp. 44 (1985), 533-535.

21. D. R. Stinson and E. Seah, 284457 Steiner triple systems of order 19 contain a subsystem of order 9, Math. Comp. 46 (1986), 717-729.

22. L. Teirlinck, On Steiner spaces, J. Combin. Theory Ser. A 26 (1979), 103-114.

(C. J. Colbourn) Department of Combinatorics and Optimization, University of WaTERloO, Waterloo, OntaRio N2L 3G1, Canada

E-mail address: cjcolbou@watmath.waterloo.edu

(S. S. Magliveras and D. R. Stinson) Department of Computer Science And Engineering, UNIVERSity OF NEBRASKA-LiNCOLN, LinCOLN, NEBRASKa 68588

E-mail address, S. S. Magliveras: spyros@helios.unl.edu

E-mail address, D. R. Stinson: stinson@bibd.unl.edu 


\title{
Supplement to \\ STEINER TRIPLE SYSTEMS OF ORDER 19 WITH NONTRIVIAL AUTOMORPHISM GROUP
}

\author{
CHARLES J. COLBOURN, SPYROS S. MAGLIVERAS, AND D. R. STINSON
}

This supplement is an exhaustive listing of all $S T S(19)$ having automorphism groups of order at least 9. We employ a very succinct representation. Each design is on the symbols $a-r$, and is represented by a string of 57 symbols $s_{1} \ldots s_{57}$. Using usual lexical order, the symbol $s_{i}$ is the largest element in the $i$ th triple; the remaining two elements of the $i$ th triple are implicitly taken to be the elements $x_{i}<y_{i}$ for which $\left\{x_{i}, y_{i}\right\}$ does not appear in an earlier triple, and there is no pair $x_{i}^{\prime}<y_{i}^{\prime}$ with this property for which $y_{i}^{\prime}$ precedes $y_{i}$, or $y_{i}=y_{i}^{\prime}$ and $x_{i}^{\prime}$ precedes $x_{i}$. We list the systems grouped by subset of basic automorphism types, and list an automorphism group order for each:

Order Triple System

$1^{7} 3^{4}, 1^{1} 3^{6}, 1^{7} 2^{6}, 1^{3} 2^{8}$

432 dofghiihjhiklmnonmpoqr smosnqpr lonrmqspprsqsprqoqreperqres

108 dofghiihghi jklmnopqrsnomrpqs lomsnqrprnsopqposqrqs

$19^{1}, 1^{7} 3^{4}$

171 defghijkllmknnmopqqrsmjksqquprplomrqupoosrnqpsorpqerrsqrss

57 defghiihgjklmnopqrprmnos lms l pponq $s$ opqrqorpsnorqpsqs $q$ qres

57 defgghijklmjnopqqkmpjrins smrnlopsrqoqrospnopqrspqsrrsqsrs $1^{1} 3^{6}, 1^{7} 2^{6}, 1^{3} 2^{8}$

144 defghiihghjjklmnopqrs lqnops rmpomqrsnqsornpposrqspqrrsqrss def ghi $j$ khk $j$ lmnomnolmpqrsnspqrooqrs pnmrsqprqpsospqrrsqses defgghi jklmnnolipqrsqsplrknpqrlsoonmrsqponsproqrsqpsqrrss defgghijklmjnopkkl qrnsqnosmormpponqmes sqsprpsqosrqpsqrsrs dofgghhijikjlmknopqonrs Imqrspnrposqmposqnrrspqospqrsrqrss defgghi $j k 1$ mmnolipqrsqsplrknpqrlsoonmrsqponsproqrsqprsqses defgghhi jikklmjnopnmqrsqolrsprnsmpqoposqrnxposqqpqrsrqrss dofgghi jklmmnolipqrspqs lrknqrplsoonms rpqonrspogsqrpsrqrss defgghhi $j i k i j l m n o p q r s$ lqsornpmorpsmqnnrsqposproqqpsrqusss defgghi $j i k k$ m jnmopqrnsqrplsoonsqprmrpoqsnsoprqs rpqsrqrs: defgghhi $j i k$ Imnopnqrkoqlpsql rosmnmsprspqornsqorppsrqq $s q x s$ defgghhijikj lmknmopqrns qrplsosnoqprmrpsqonosprqspqrsrqrss defgghi jklmjnopkl jmqnrpsoqrnosmmnogsrppsrqsprqarqgpqsirss defgghhi jklmnomplqnrnlrsmqkspqrolopssropnqqopsrrqsprqssrs defgghhi jkli jmnopqqr snkorpsmlmpsnqronqorspporsqpqsrsrqgrs defgghhi jklmnojplqnrlrsmqspqrolmnopsnsropqqopsrrqsprqssrs dof gghhi jkli jmnopjqrsorpsmlqnmpsnqronqorspporqspqsrsrqgss

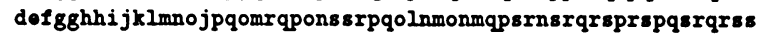
def gghhi jkli jmnopqqrsnkorpsmlmnqorsppsnqroporsqpqsrsrqs $r$ dof gghhi jklmnojplqnrlrsmpsqprolmnoqsns roqpqopsrrpsqgrqq 88 defgghhijkli jmnopjqrsorpsmlqnmnqorsppsnqroporqgsqsrsrqsrs $1^{7} 3^{4}, 1^{1} 3^{6}, 1^{7} 2^{6}$

54 defggthijklmnolnpkqrrnkslqomqpsposmrqornspsrpoqqpsrrqssrs 54 defggthijklmnomopqlrmlrospkpqsornnqpsmsrnqoprsqqsrpsqriss 18 dofggthijklmnolknmojopqrs spqlrmrqpsnqrsnpops rqoqpsrqgrrss

18 defggthijklmnolknmojopqrsspqlrmrqpsnqsinpoprsqoqpsrqsirss 

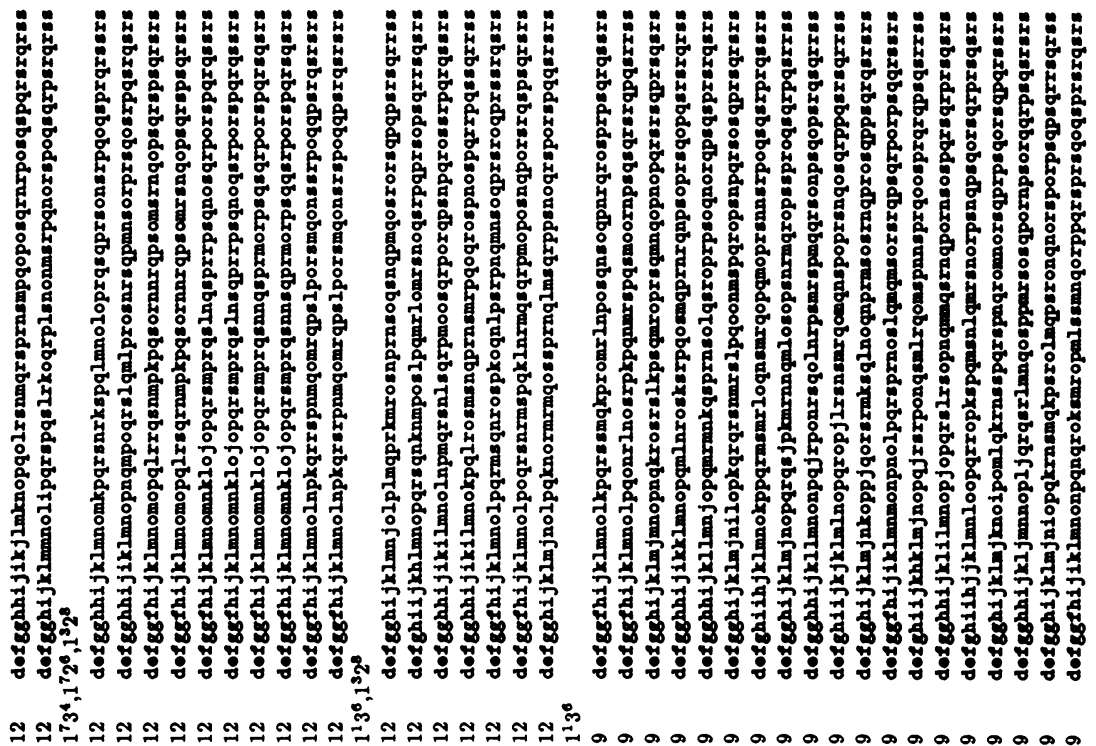
ำ

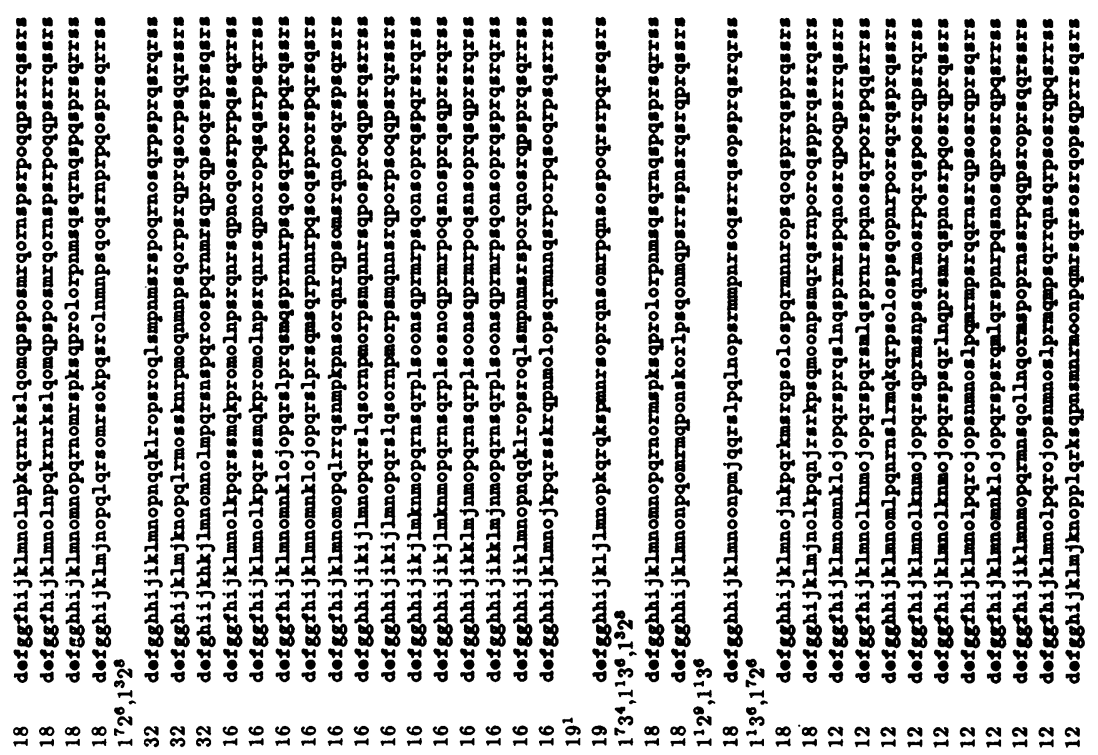




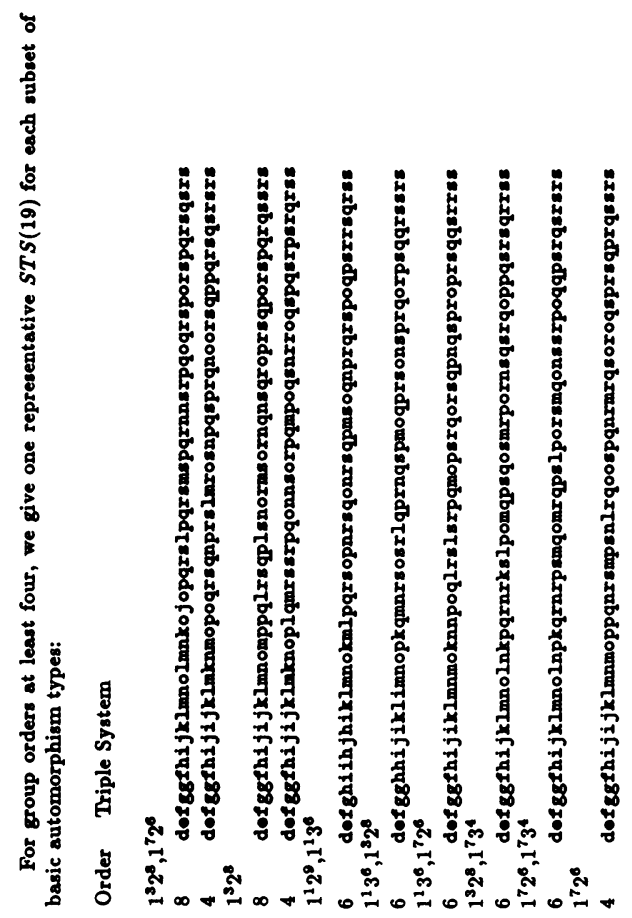

